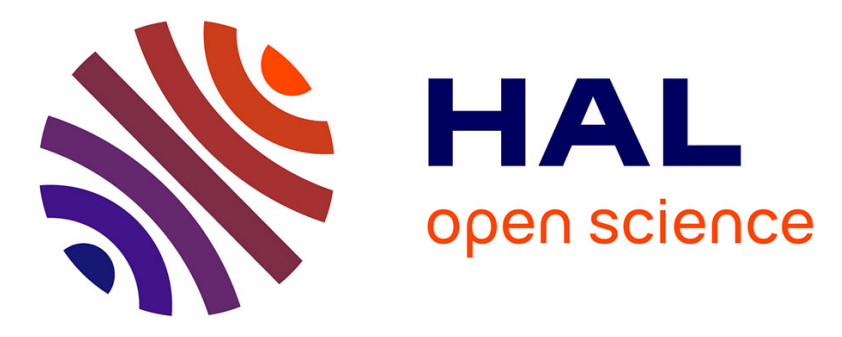

\title{
Measuring noise and cross correlations at high frequencies in nanophysics
}

\author{
Marjorie Creux, A. Zazunov, Thi Kim Thanh Nguyen, Adeline Crépieux, \\ Thierry Martin
}

\section{- To cite this version:}

Marjorie Creux, A. Zazunov, Thi Kim Thanh Nguyen, Adeline Crépieux, Thierry Martin. Measuring noise and cross correlations at high frequencies in nanophysics. International Symposium on Mesoscopic Superconductivity and Spintronics 2006 (MS+S2006), Feb 2006, Atsugi, Japan. pp.359. hal-00479501

\section{HAL Id: hal-00479501 https://hal.science/hal-00479501}

Submitted on 30 Apr 2010

HAL is a multi-disciplinary open access archive for the deposit and dissemination of scientific research documents, whether they are published or not. The documents may come from teaching and research institutions in France or abroad, or from public or private research centers.
L'archive ouverte pluridisciplinaire HAL, est destinée au dépôt et à la diffusion de documents scientifiques de niveau recherche, publiés ou non, émanant des établissements d'enseignement et de recherche français ou étrangers, des laboratoires publics ou privés. 


\title{
MEASURING NOISE AND CROSS CORRELATIONS AT HIGH FREQUENCIES IN NANOPHYSICS
}

\author{
M. CREUX, A. ZAZUNOV, T.K.T. NGUYEN, A. CRÉPIEUX, AND T. MARTIN \\ Centre de Physique Théorique et Université de la Méditerranée \\ Case 907, 13288 Marseille, France
}

\begin{abstract}
The purpose of the present paper is to propose two scenarios for measuring high frequency noise. The first one uses inductive coupling to an LC circuit, and describes the measurement of noise cross-correlations as in an Hanbury-Brown and Twiss geometry. The second one uses the photo-assisted transfer of two electrons in a normal metalsuperconductor circuit, which is capacitively coupled to the mesoscopic circuit to be measured.
\end{abstract}

\section{Introduction}

The measurement of finite frequency noise in mesoscopic systems provides a crucial diagnosis of the carriers involved in the transport ${ }^{1,2}$. Here, we are interested in 2 types of systems where noise is detected via a measuring device - coupled to the mesoscopic device which should pick up (via repeated time measurements) the noise contribution at a specific frequency, without the manipulation of a time series. Such proposals have been put forth and some have been implemented experimentally within the last decade ${ }^{3,4,5,6,7,8}$. Low frequency noise cross-correlations measurements have also been performed ${ }^{9,10}$, which showed that a fully degenerate electron gas has negative noise correlations. Yet, finite frequency noise cross-correlations are useful in the study of electronic entanglement in mesoscopic devices ${ }^{11}$, and in the identification of anomalous charges in Luttinger liquid wires ${ }^{12}$. The purpose of the present paper is twofold: to suggest a way to measure noise cross-correlation with inductive coupling, and to suggest a way to measure noise via photo-assisted Andreev reflection in a capacitive coupling setup.

\section{Measurement of noise cross-correlations with inducting coupling}

For measuring cross-correlations with an LC circuit, two inductances $\left(L_{1}\right.$ and $\left.L_{2}\right)$ and a single capacitor $(C)$ are needed. The two inductors, with coupling constants $\alpha_{1}$ and $\alpha_{2}$, are placed next to the two outgoing arms of the three terminal mesoscopic device (Fig. 1), and is placed in series. Depending on the wiring of these inductances (Fig. 1a or Fig. 1b), the two inductances "see" the outgoing currents with the opposite sign or with the same sign. 


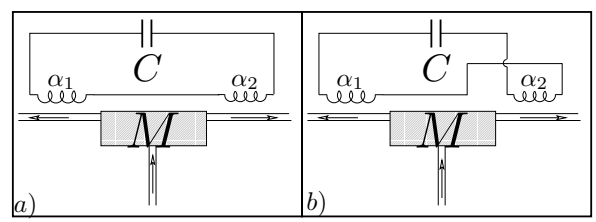

Figure 1. Schematic description of the noise cross-correlation setup. $M$ is the mesoscopic circuit to be measured, $C$ is the capacitor and there are two inductors with coupling constants $\alpha_{1}$ and $\alpha_{2}$ to the mesoscopic circuit. The electrical components of the detector are in series and they "see" the current with the same sign $(a)$ or with the opposite sign $(b)$.

It was shown ${ }^{4}$ that finite frequency noise can be detected by coupling inductively an LC circuit to the wires connected to an arbitrary mesoscopic device. Here we address two issues: a) we generalize this proposal to the measurement of noise cross correlations; b) we briefly mention the role played by dissipation in this measurement scheme. In the initial proposal ${ }^{4}$, the noise is measured at the resonant frequency of the LC circuit, by performing repeated time measurements of the charge fluctuations on the capacitor plates. The measured noise turns out to be a mixture of the two unsymmetrized noise correlators, which correspond to emission and absorption of radiation from the mesoscopic device.

For the case of cross correlations, one measures the charge fluctuations $\left\langle x^{2}(0)\right\rangle_{2+}$ with the geometry of Fig. 1a and subsequently one can switch the wiring and measure such fluctuations $\left\langle x^{2}(0)\right\rangle_{2-}$ with the circuit of Fig. 1b. By subtracting the two signals ${ }^{13}$ :

$$
\left\langle x^{2}(0)\right\rangle_{2}=\frac{1}{2}\left(\left\langle x^{2}(0)\right\rangle_{2+}-\left\langle x^{2}(0)\right\rangle_{2-}\right)
$$

one isolates the contribution of cross-correlations, which is proportional to $\alpha_{1} \alpha_{2}$. The following non-symmetrized current correlators are introduced in Fourier space:

$$
S_{i j}^{+}(\omega)=\int \frac{d t}{2 \pi} e^{i \omega t}\left\langle I_{i}(0) I_{j}(t)\right\rangle, \quad S_{i j}^{-}(\omega)=\int \frac{d t}{2 \pi} e^{i \omega t}\left\langle I_{i}(t) I_{j}(0)\right\rangle .
$$

with $i, j=1,2$ corresponding to the leads of the mesoscopic circuit. The charge fluctuations take the final form:

$$
\left\langle x^{2}(0)\right\rangle=\frac{\pi \alpha_{1} \alpha_{2}}{\eta(2 M)^{2}} \operatorname{Re}\left[(N(\Omega)+1) S_{12}^{+}(\Omega)-N(\Omega) S_{12}^{-}(\Omega)\right]
$$

with $N(\Omega)=1 /\left(e^{\beta \hbar \Omega}+1\right)$ is the Bose Einstein distribution at the detector circuit temperature, which is not necessarily the mesoscopic device temperature, and $M=$ $L_{1}+L_{2}$ is the "mass" of the detector circuit. Note the presence of the adiabatic parameter $\eta$, which suggests that the measured noise diverges! If one includes dissipation in the $\mathrm{LC}$ circuit due to a finite resitance of the circuit using the CaldeiraLegett model ${ }^{14}$, it turns out ${ }^{15}$ that $\eta$ is replaced by the linewidth of the LC circuit.

Next, this proposal is tested on a system of three terminals (Fig. 2), a so called "Y junction", described by scattering theory. We consider the case where the voltage biases satisfy $\mu_{13}>\mu_{12}>\mu_{23}>0\left(\mu_{i j} \equiv \mu_{i}-\mu_{j}\right)$. At a low temperature, the charge 
fluctuations stay negative and singularities are present at frequencies equal to $\mu_{23}$, $\mu_{12}, \mu_{13}$. When the temperature becomes larger than the bias voltages, the charge fluctuations become positive. However, when the temperature goes to zero, the measured charge fluctuations become equal to $S_{23}^{+}(\omega)$.

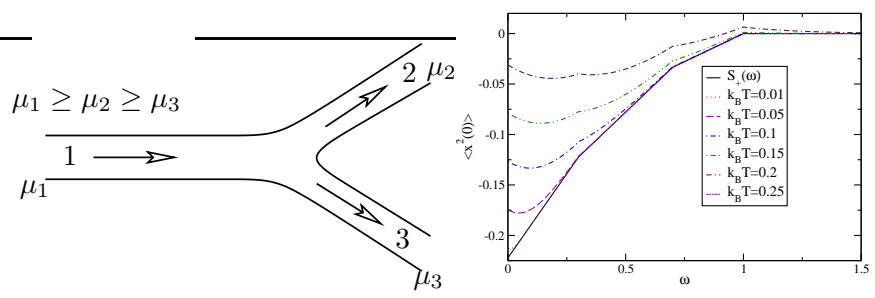

Figure 2. In the left, a system with three terminals (Y junction) with chemical potentials $\mu_{1}, \mu_{2}$ and $\mu_{3}$ with $\mu_{1} \geq \mu_{2} \geq \mu_{3}$. In the right, measured charge fluctuations as a function of frequency for different temperatures $k_{B} T$, "measured" in units of $\mu_{13}$. The frequency and the biases are in units of $\mu_{13} . \mu_{12}=0.7 \mu_{13}, \mu_{23}=0.3 \mu_{13}$ have been chosen.

\section{Noise measurement via photo-assisted Andreev reflection}

A high frequency noise measurement setup was proposed, which used capacitive coupling ${ }^{5}$ between a double quantum dot and a nearby mesoscopic conductor. This idea was implemented recently ${ }^{6}$ using a superconductor-insulator-superconductor (SIS) junction for the detector circuit: a DC measurement of quasiparticle tunneling provided information on high frequency noise. The purpose of the present work
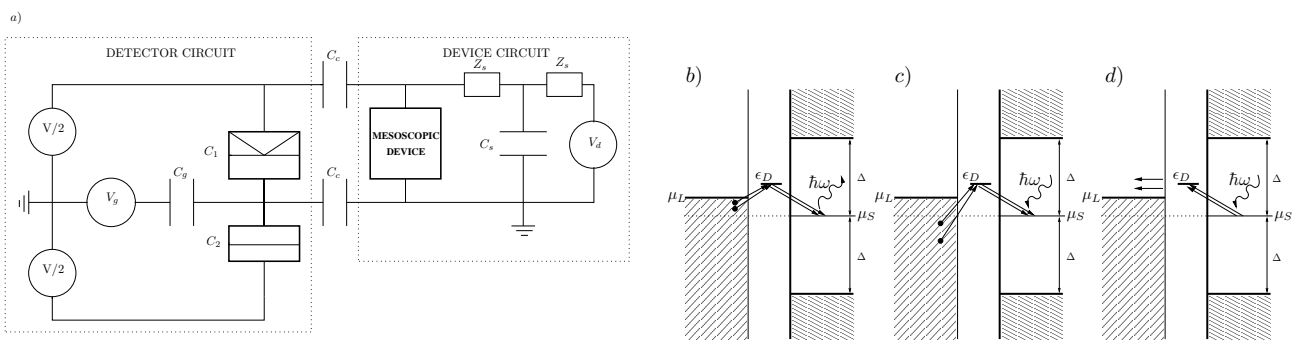

Figure 3. a) Schematic description of the set up: the mesoscopic device to be measured is coupled capacitively to the detector circuit. The latter consists of a normal metal lead-quantum dotsuperconductor circuit with a DC bias. b), c), d) Photo-assisted Andreev reflection: b), c) Emission of a Cooper pair in a normal metal in the case of a "photon" is provided to or provided by a neighboring environment. d) Case of absorption of a Cooper pair with photo-assisted Andreev reflection, where a "photon" is provided by a neighboring environment. Notice that the tunneling of electrons is sequential.

is to analyze a similar situation, except that the SIS junction is replaced by a circuit which transfers two electrons via Andreev reflection. Here, Andreev reflection 
processes occur through a quantum dot, allowing to filter electron energies: two inelastic electron jumps are required for a current to pass through the measurement circuit, the energy being injected/emitted by a "photon" provided/absorbed by the mesoscopic circuit to be tested. The circuit is depicted in Fig. 3a. The charging energy of the quantum dot is large enough that double occupancy is prohibited. This dot is in tunneling contact with a superconductor. Two capacitors are placed between each side of the mesoscopic device and each side of the quantum dotsuperconductor junction. Current fluctuations in the mesoscopic device generate voltage fluctuations across the dot-superconductor tunnel junction, which translate into phase fluctuations at the junction, in the spirit of the dynamical Coulomb blockade ${ }^{16}$.

The Hamiltonian which describes the decoupled normal metal lead-dotsuperconductor-environment (mesoscopic circuit) system reads

$$
H_{0}=\sum_{k, \sigma} \epsilon_{k} c_{k, \sigma}^{+} c_{k, \sigma}+\sum_{\sigma} \epsilon_{D} c_{D, \sigma}^{+} c_{D, \sigma}+U n_{\uparrow} n_{\downarrow}+\sum_{q, \sigma} E_{q} \gamma_{q, \sigma}^{+} \gamma_{q, \sigma}+\mu_{S} N_{S}+H_{e n v}
$$

which correspond to the normal metal lead, the quantum dot (single occupancy only), and the (BCS) superconductor, described in terms of quasiparticle operators $\left(\gamma_{q, \sigma}\right)$ which are related to $c_{q, \sigma}$ by the Bogoliubov transformation. The tunneling Hamiltonian reads:

$$
H_{T}=\sum_{q, \sigma} T_{D, q} c_{D, \sigma}^{+} c_{q, \sigma} e^{-i \phi}+\sum_{k, \sigma} T_{k, D} c_{k, \sigma}^{+} c_{D, \sigma}
$$

The fluctuating phase factor represents the coupling to the mesoscopic circuit. All the information about the environment is contained in the autocorrelation of the phase operators $J(t)=\langle[\phi(t)-\phi(0)] \phi(0)\rangle$. The phase operator is related to the voltage by $\phi(t)=e \int_{-\infty}^{t} d t^{\prime} V\left(t^{\prime}\right)$. Indeed, because of the capacitive coupling between the sides of the dot-superconductor junction and the mesoscopic circuit, current fluctuations of the mesoscopic device translate into voltage fluctuations of the dotsuperconductor junction across the trans-impedance ${ }^{5} \delta V(\omega)=Z(\omega) \delta I(\omega)$.

The current through the normal lead/quantum dot/superconductor (NDS) junction, due to the tunneling of two electrons, is calculated using the Fermi Golden Rule generalized with the T-matrix. Here one needs to carry out calculations of the matrix element to fourth order in the tunneling Hamiltonian in order to describe electron transfer. The inelastic current through the NDS detector reads:

$$
\begin{aligned}
I^{\text {inel }} \simeq & \frac{16 \pi^{2} e}{R_{K}} \mathcal{N}_{N}^{4} T_{1}^{4} T_{2}^{4} \int_{\Delta}^{\infty} d E \int_{\Delta}^{\infty} d E^{\prime} \frac{\Delta^{2}}{\sqrt{E^{2}-\Delta^{2}} \sqrt{E^{\prime 2}-\Delta^{2}}} \\
\times & \left\{\int_{-\infty}^{e V} d \epsilon \int_{-\infty}^{e V} d \epsilon^{\prime} \frac{\left|Z\left(\epsilon+\epsilon^{\prime}\right)\right|^{2}}{\left(\epsilon+\epsilon^{\prime}\right)^{2}} \frac{S_{I}\left(\epsilon+\epsilon^{\prime}\right)}{D^{\text {inel }}}\right. \\
& \left.\quad-\int_{e V}^{\infty} d \epsilon \int_{e V}^{\infty} d \epsilon^{\prime} \frac{\left|Z\left(-\left(\epsilon+\epsilon^{\prime}\right)\right)\right|^{2}}{\left(\epsilon+\epsilon^{\prime}\right)^{2}} \frac{S_{I}\left(-\left(\epsilon+\epsilon^{\prime}\right)\right)}{D^{\text {inel }}}\right\}
\end{aligned}
$$

where $D^{\text {inel }}$ is an energy denominator product ${ }^{17}, R_{K}=2 \pi / e^{2}$ is the quantum of resistance, $Z(\omega)$ is the trans-impedance of the circuit and $S_{I}(\omega)=S^{+}(-\omega)$, with 

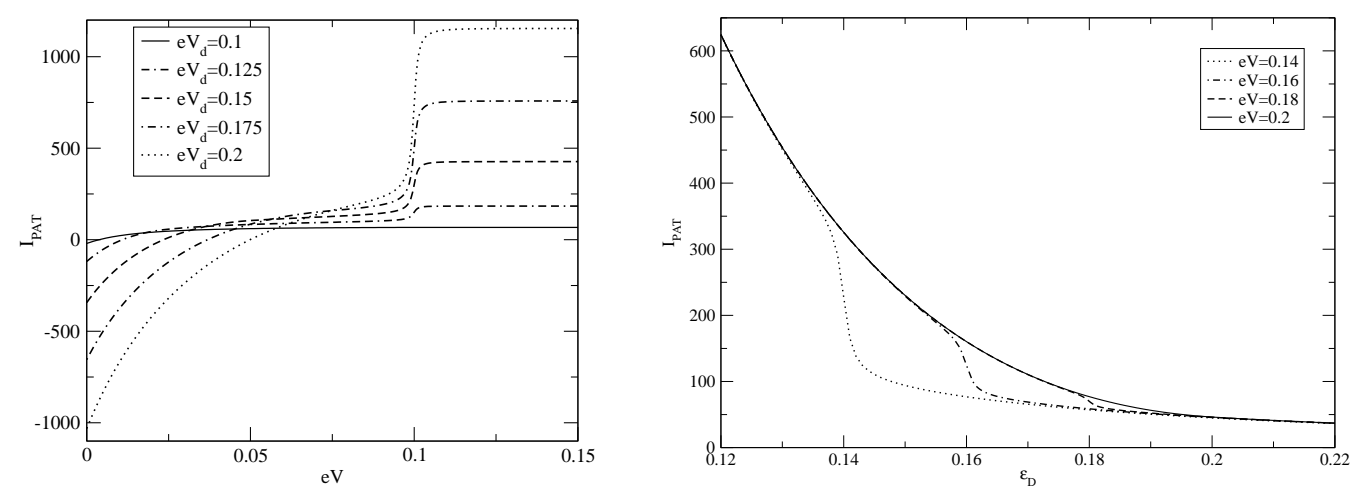

Figure 4. Left pannel: PAT current plotted as a function of detector bias voltage for some values of the device bias voltage $e V_{d}$ from 0.1 to 0.2 (see legend), with $\epsilon_{D}=0.1$. Right pannel: PAT current depends on dot level $\epsilon_{D}$ with some values of detector bias voltage $e V: 0.14,0.16,0.18,0.2$ (see legend) at $e V_{d}=0.2$.

$S^{+}(\omega)=\int d t e^{i \omega t}\langle\langle I(0) I(t)\rangle\rangle$. The first term in Eq. (6) describes the tunneling of a Cooper pair from the normal lead to the superconductor via the quantum dot: as depicted in Figs. 3b,c the tunneling between the dot and the superconductor involves the emission/absorption of a photon. The second term describes the inverse tunneling process: a Cooper pair absorbing energy from the neighboring device (Figs. 3d), its constituent electrons then tunneling from the superconductor to the normal lead.

We illustrate the present results by considering a point contact for the mesoscopic circuit. We compute the photon assisted tunneling (PAT) current through the detector due to the high frequency current fluctuations of the device, as a function of detector bias voltage $I_{P A T}(e V)=I\left(e V_{d} \neq 0, e V\right)-I\left(e V_{d}=0, e V\right)$ (excess current), at zero temperature for simplicity. The left panel of Fig. 4 depicts the PAT current as a function of detector bias voltage for a fixed dot energy level position, but for different values of $e V_{d}$. When the bias of the mesoscopic device increases, the noise increases, energy emitted or absorbed also increases, which triggers the PAT current. ¿From $e V_{d}=\epsilon_{D}$, if we decrease $e V_{d}$, the PAT current is reduced, approaching 0 and the step disappears hereafter. This is realized at the point when the spectral density of noise of the mesoscopic device (point contact) contains a singularity in its derivative.

In the right panel of Fig. 4, we plot the dependence of the PAT current on the dot energy level for several values of the bias voltage of the detector circuit, which is chosen smaller than the device bias voltage $e V_{d}=0.2$, specifying $e V>$ $e V_{d} / 2$. By choosing this range of $e V$, only the current from the normal metal to the superconductor contributes: the dominant contribution comes from emission processes. The PAT current decreases when the dot level is raised, however, it displays a step at $\epsilon_{D}=e V$, provided that $e V<e V_{d}$. At the step location, the PAT 
current drops fast, then it decreases more slowly and saturates. For $e V>e V_{d} / 2$, if we increase $e V$, the step height decreases and eventually vanishes when $e V=e V_{d}$. Hereafter, if one continues to increase $e V$, the PAT current is still the same as it with $e V=e V_{d}$ because the energy emission to the mesoscopic device reaches saturation.

\section{Conclusion}

We have shown how a careful wiring of the detector circuit can isolate the noise cross-correlations signal. This promises to have applications in the detection of fractional charges in carbon nanotubes ${ }^{12}$ as well as in the detection of electronic entanglement ${ }^{18}$.

For capacitive coupling to an NS circuit, by controlling the detector circuit (varying $\mathrm{eV}$ and $\epsilon_{D}$ ), we can make a mapping of the spectral density of noise. Here the frequency $\omega=e V_{d}$ corresponds to the point where the excess noise of the point contact contains a singularity in its derivative. This constitutes an encouraging scenario for detecting specific features in the noise spectrum.

\section{References}

1. C. Chamon, D. E. Freed, and X. G. Wen, Phys. Rev. B 53, 4033 (1996).

2. T. Martin in Les Houches Summer School session LXXXI, edited by E. Akkermans, H. Bouchiat, S. Gueron, and G. Montambaux (Springer, 2005).

3. R. J. Schoelkopf et al., Phys. Rev. Lett. 78, 3370 (1997). J. Gabelli et al., Phys. Rev. Lett. 93, 056801 (2004).

4. G. B. Lesovik and R. Loosen, Pis'ma Zh. Éksp. Teor. Fiz. 65, 280 (1997) [JETP Lett. 65, 295, (1997)]; Y. Gavish, I. Imry, and Y. Levinson, Phys. Rev. B 62, 10637 (2000).

5. R. Aguado and L. Kouwenhoven, Phys. Rev. Lett. 84, 1986 (2000).

6. R. Deblock et al., Science 301, 203 (2003); A. Onac et al.,Phys. Rev. Lett. 96, 026803 (2006); P.-M. Billangeon et al., cond-mat/0508676.

7. R. Deblock, E. Onac, L. Gurevich, and L. P. Kouvenhoven, Science 301, 203 (2003).

8. P.-M. Billangeon, F.Pierre, H. Bouchiat, and R. Deblock, cond-mat/0508676.

9. T. Martin and R. Landauer, Phys. Rev. B 45, 1742 (1992); M. Büttiker, Phys. Rev. B 45, 3807 (1992).

10. M. Henny, Science 284, 296 (1999); W. D. Oliver et al., Science 284, 299 (1999).

11. G. B. Lesovik, T. Martin, and G. Blatter, Eur. Phys. J. B. 24, 287 (2001); P. Recher, E.V. Sukhorukov, and D. Loss, Phys. Rev. B 63, 165314 (2001).

12. A. V. Lebedev, A. Crépieux, and T. Martin, Phys. Rev. B 71, 075416 (2005).

13. M. Creux, A. Crépieux, and T. Martin, cond-mat/0507708.

14. A.O. Caldeira and A.J. Leggett, Phys. Rev. A 311059 (1985)

15. M. Creux, A. Zazunov, E. Paladino, A. Crépieux, and T. Martin, in preparation.

16. G. L. Ingold and Yu. V. Nazarov, in Single Charge Tunneling, H. Grabert and M.H. Devoret eds. (Plenum, New York 1992).

17. T. Nguyen et al., cond-mat/0602408.

18. N. Chtchelkatchev et al., Phys. Rev. B 66, 161320 (2002). 